\title{
Production Lot Sizing and Process Targeting under Process Deterioration and Machine Breakdown Conditions
}

\author{
Muhammad Al-Salamah ${ }^{1}$ and Ali Abudari \\ ${ }^{1}$ Mechanical Engineering Department, College of Engineering, Majmaah University, Majmaah 11952, Saudi Arabia \\ ${ }^{2}$ Business Improvement Department, Rezayat Group, P.O. Box 90, Khobar 31952, Saudi Arabia
}

Correspondence should be addressed to Muhammad Al-Salamah, m.alsalamah@mu.edu.sa

Received 3 September 2011; Revised 28 October 2011; Accepted 30 October 2011

Academic Editor: Jing-song Hong

Copyright ( $\odot 2012$ M. Al-Salamah and A. Abudari. This is an open access article distributed under the Creative Commons Attribution License, which permits unrestricted use, distribution, and reproduction in any medium, provided the original work is properly cited.

The paper considers a production facility that might deteriorate suddenly at some point during the production run time; after deterioration, nonconforming items are produced in a greater rate compared to the rate before deterioration. Moreover, the production facility may ultimately break down; consequently, the production lot is aborted before completion. If breakdown happens, corrective action is started immediately; otherwise, the production lot is completed and preventive repair is implemented at the end of the production cycle to enhance system reliability. The mathematical model is formulated under general distributions of failure, corrective, and repair times, while the numerical examples are solved under exponential failure and uniform repair times. The formulated model successfully determines the optimal lot size in addition to the optimal process parameters (mean and standard deviation) simultaneously.

\section{Introduction}

In real-life situations, most production systems are unreliable to a significant degree, and process deterioration occurs at some point in the production run time. A process shift from in-control state to out-of-control state will cause the production of more nonconforming items. This process may go worse and ultimately machine breakdown happens causing production interruption, and consequently plans for meeting demand are severely affected. Building on the previous argument, the need for more realistic modeling of EMQ (Economic Manufacturing Quantity) is rising in the manufacturing field; such modeling should take into consideration system variables which affect performance in a random manner; some of those variables include the following.

(1) Time to shift from in-control state to out-of control state.

(2) Time to breakdown.

(3) Corrective maintenance time.

(4) Preventive maintenance time.
(5) Rate of producing nonconforming items before and after process shift; this can be handled in various ways.

(a) Assuming nonconforming items are produced only after deterioration and they are produced at constant rate.

(b) Assuming nonconforming items are produced before and after a sudden deterioration; accordingly, the process mean shifts to a new value and stays unchanged. The rate of producing nonconforming items is obviously greater after deterioration, and in both cases, the rate depends on the value of the process mean.

(c) Or deterioration might be considered to happen gradually following a specific pattern (e.g., linear, quadratic, or exponential).

(6) Usual costs related to maintenance, production, and inventory such as corrective and preventive repair costs, inventory holding cost, shortage penalty cost, cost of producing nonconforming items, and set-up cost. 
Obviously, the two factors that have a great influence on EMQ decisions are process deterioration and machine breakdown. During the last few years, researchers from all over the world have done a tremendous amount of work investigating those two issues and their effect on EMQ.

Dagpunar [1] examined the lot-sizing problem with machine time to failure following a Weibull distribution; the machine is minimally repaired until the interrupted lot is completed; at the end of the production cycle, the machine is restored to as-good-as-new condition and new cycle is started. Kuhn [2] suggested a stochastic dynamic programming model to determine the optimal lot sizing decision where the equipment is subject to stochastic breakdowns. The analysis considered two cases; first it is assumed that, after the machine breakdown, the setup is totally lost and new setup cost is incurred; the second case considers the situation in which the cost of resuming the production run after a failure might be substantially lower than the production set-up cost. Ben-Daya [3] dealt with an integrated model for the joint determination of economic production quantity and preventive maintenance (PM) level for an imperfect process having a general deterioration distribution with increasing hazard rate; the effect of PM activities on the deterioration pattern of the process is modeled using the imperfect maintenance concept. Chung [4] showed that the long-run average cost function per unit of time for the case of exponential breakdowns is unimodal but neither convex nor concave, and he obtained an approximation for lower and upper bounds on lot sizing under this condition.

Giri and Dohi [5] considered the net present value (NPV) approach to determine the economic manufacturing quantities for an unreliable production system over an infinite planning horizon. The NPV of the expected total cost was obtained under general breakdown time and general repair time. The criteria for the existence and uniqueness of the optimal production time (lot size) were derived under exponential breakdown and constant/zero repair time.

Giri and Yun [6] considered an economic manufacturing quantity problem for an unreliable manufacturing system where the machine is subject to random breakdown and at most two failures can occur in a production cycle. Upon first failure, the repair action is started immediately and the demand is met first from the on-hand inventory. The shortages, if they occur due to longer repair time, are backlogged partially by resuming the production run after machine repair. While backlogging, if failure occurs again then the accumulated shortages until completion of the second repair are assumed to be lost. The model was formulated under general breakdown and general repair time distributions.

Hsieh and Lee [7] considered two economic manufacturing quantity models with unrepairable and repairable standby key modules. They determined the economic production run length and the economic number of standbys in a deteriorating production process. Jaber [8] investigated the lot-sizing problem for reduction in setups, with reworks, and interruptions to restore process quality after process deterioration takes place. He assumed the rate of producing defectives to benefit from any changes for eliminating the defects and thus reduces with each quality restoration action.
Chiu et al. [9] studied the optimal lot-sizing decision for a production system with rework, random scrap rate and service level constraint. El-Ferik [10] studied the joint determination of both economic production quantity and preventive maintenance (PM) schedules under the realistic assumption that the production facility is subject to random breakdown and the maintenance is imperfect. The manufacturing system was assumed to deteriorate while in operation, with an increasing failure rate. The system undergoes PM either upon failure or after having reached a predetermined age, whichever of them occurs first. Chelbi et al. [11] proposed an integrated production-maintenance model for unreliable production system producing conforming and nonconforming items. The model is designed to simultaneously determine the optimal values of the lot size and the age at which preventive maintenance must be performed in order to minimize the total cost.

Chakraborty et al. [12] developed integrated production, inventory, and maintenance models for a deteriorating production system in which the production facility may shift from an "in-control" state to an "out-of-control" state and break down at any random point in time during a production run. In case of machine breakdown, production of the interrupted lot is aborted and a new production lot is started when the on-hand inventory is depleted and after corrective repair is completed. The process is inspected during each production run to examine the state of the production process.

Pentico et al. [13] investigated EOQ with partial and full backordering production systems. Hu and Zong [14] proposed an extended product inspection policy for a deteriorating production system. Based on their model, they showed that there exists a production run time and a corresponding unique inspection policy such that the expected total cost per item per cycle is minimized.

Up to our knowledge, no one has considered process targeting and production lot sizing simultaneously under process deterioration and machine breakdown conditions. In this paper we are going to study the joint effect of process deterioration, machine breakdown, and random repair times (corrective and preventive) on the optimal lot-sizing decisions in addition to process parameters (mean and standard deviation). We will consider process deterioration to happen suddenly, meaning that the process mean immediately shifts from original value $\mu_{I}$ to a new value $\mu_{O}$.

While determining the EMQ (or production run time) is important for production planning, one can question the economic benefits that can be drawn from a model that aims to find the optimal values of the process parameters: mean and standard deviation. For a quality characteristic, the performance of the process is quantified using the specification limits, lower specification limit (LSL) and upper specification limit (USL). The cost of producing items within the accepted range, LSL $<x<$ USL, is evaluated by the Taguchi loss function which is dependent on the process mean. Also the cost associated with producing items out of the specification limits is entirely affected by the process mean. Process targeting problems are found in practical situations such as filling problems where one has to control the overfilling 
and underfilling costs by finding the optimal process mean which lies within the specification limits. On the other hand, the process standard deviation reflects the machine's accuracy and precision. A lower standard deviation means more expensive machines with higher accuracy levels. Hence, finding the optimal value for the standard deviation which can achieve the lowest cost is essential in minimizing the overall cost of the process.

\section{Model Formulation}

The model under consideration is describing a production facility which may shift from in-control-state to out-ofcontrol state at any random time during a production run; in both states, nonconforming items are produced in different rates depending on the process mean value. The production facility may also break down at any time during a production cycle. Once a shift to the out-of-control state has occurred, it is assumed that the production process will stay in that state until the whole lot has been produced or machine breakdown occurs. If the machine breakdown happens during the production run, then the interrupted lot is aborted and a new lot is started after corrective maintenance when all available inventory is depleted (no resumption-NR-policy).

Assuming that the production process starts in an "incontrol" state at time $t=0$ to produce an amount $Q$, if the machine breakdown does not occur until the time $t_{o}=Q / p$, then preventive maintenance (PM) is carried out to bring the machine to the as-good-as-new condition before the start of the next production run. However, if the machine breaks down before producing the required quantity $Q$, then the corrective repair action is started immediately. During the machine repair (corrective and preventive), the demand is met first from the accumulated on-hand inventory. Shortages may occur due to longer corrective/preventive repair times. If shortages occur, they are not delivered after the machine repair.

Figures 1-4 show all possible scenarios during a production cycle. In Figure 1, machine failure happens at time $t<t_{0}$; consequently, the production lot is aborted and corrective repair is started immediately and completed before inventory is totally depleted causing no shortages. Figure 2 shows the case where machine failure happens at time $t<t_{0}$, and shortages occur due to long corrective repair time. In Figures 3 and 4 , no machine failure happens, accordingly preventive repair started immediately at the end of the production cycle, and again shortages may occur due to longer repair times.

2.1. Lot Sizing and Targeting Process Mean. When deterioration happens after time $\tau$, process mean immediately shifts from $\mu_{I}$ to $\mu_{O}$. The in-control and out-of-control process means are assumed to be related as in the following equation:

$$
\mu_{O}=\gamma \mu_{I}, \quad \gamma>0 .
$$

The probability density functions for the quality characteristic $X$ before and after process shift are $q\left(x, \mu_{I}\right)$ and $q\left(x, \mu_{O}\right)$, respectively, and they are assumed to be normal

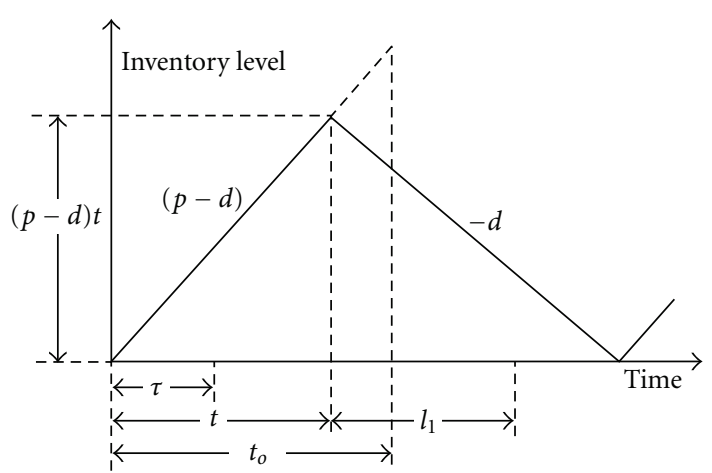

FIgURE 1: Breakdown/no shortage.

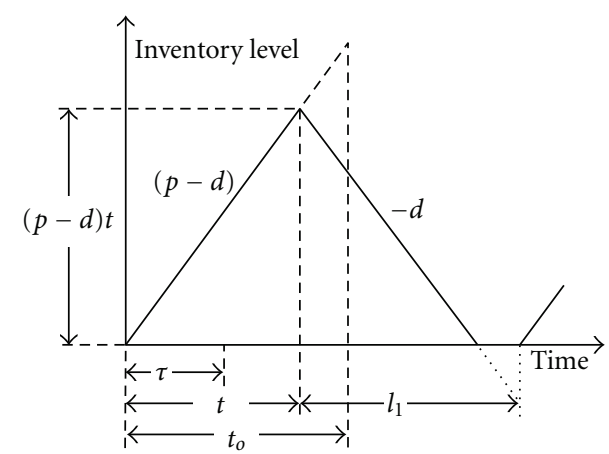

FIgURE 2: Breakdown/shortage.

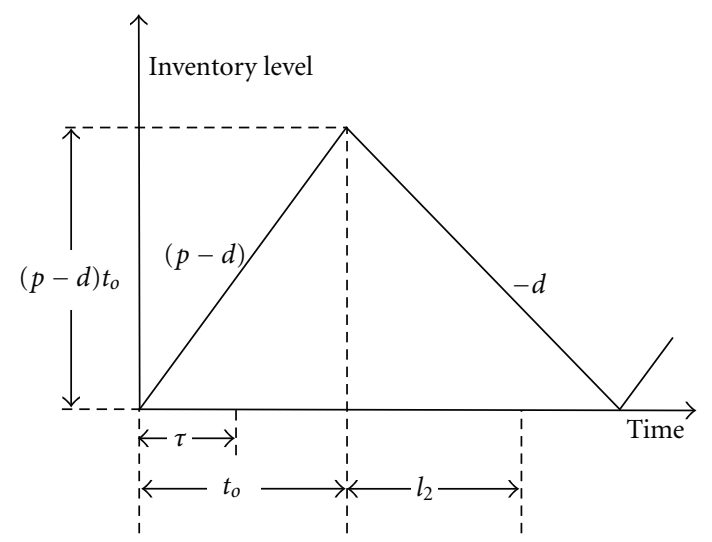

FIgURE 3: No Breakdown/no shortage.

with known and constant standard deviation $\sigma$. The fraction nonconforming before and after the process shift is given by:

$$
\begin{aligned}
& \alpha=\alpha_{1}+\alpha_{2}=\int_{x=0}^{x=\mathrm{LSL}} q\left(x, \mu_{I}\right) d x+\int_{x=\mathrm{USL}}^{x=\infty} q\left(x, \mu_{I}\right) d x, \\
& \beta=\beta_{1}+\beta_{2}=\int_{x=0}^{x=\mathrm{LSL}} q\left(x, \mu_{O}\right) d x+\int_{x=\mathrm{USL}}^{x=\infty} q\left(x, \mu_{O}\right) d x .
\end{aligned}
$$




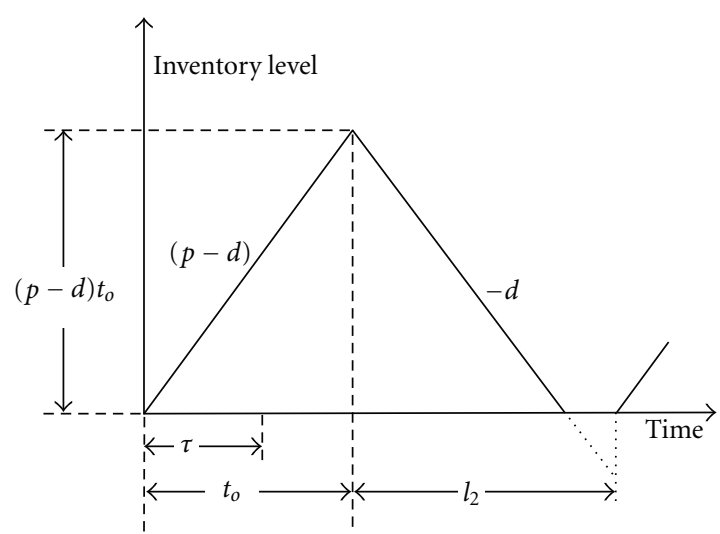

Figure 4: No Breakdown/shortage.

The expected time while the process is in-control is given by

$$
\begin{aligned}
T_{\text {in }}= & \int_{t=0}^{t_{o}} \int_{\tau=0}^{t} \tau \cdot h(\tau) \cdot f(t) \cdot d \tau \cdot d t \\
& +\int_{t=t_{o}}^{\infty} \int_{\tau=0}^{t_{o}} \tau \cdot h(\tau) \cdot f(t) \cdot d \tau \cdot d t .
\end{aligned}
$$

The expected time while the process is out-of-control is given by

$$
\begin{aligned}
T_{\text {out }}= & \int_{t=0}^{t_{o}} \int_{\tau=0}^{t}(t-\tau) \cdot h(\tau) \cdot f(t) \cdot d \tau \cdot d t \\
& +\int_{t=t_{o}}^{\infty} \int_{\tau=0}^{t_{o}}\left(t_{o}-\tau\right) \cdot h(\tau) \cdot f(t) \cdot d \tau \cdot d t .
\end{aligned}
$$

The total cost of nonconforming items in a production cycle is given by

$$
C_{N}=p \cdot T_{\text {in }} \cdot\left[c_{L} \alpha_{1}+c_{U} \alpha_{2}\right]+p \cdot T_{\text {out }} \cdot\left[c_{L} \beta_{1}+c_{U} \beta_{2}\right] .
$$

We quantify the impact of imprecision on the quality characteristic $X$ by using Taguchi loss function:

$$
L(x)= \begin{cases}\frac{c_{L}\left(x-\mu_{I}\right)^{2}}{\left(\mathrm{LSL}-\mu_{I}\right)^{2}}, & \text { if } \mathrm{LSL} \leq x \leq \mu_{I}, \\ \frac{c_{U}\left(x-\mu_{I}\right)^{2}}{\left(\mathrm{USL}-\mu_{I}\right)^{2}}, & \text { if } \mu_{I} \leq x \leq \mathrm{USL} .\end{cases}
$$

The total loss cost in a production cycle is given by

$$
\begin{aligned}
C_{\text {Loss }}=p \cdot T_{\text {in }} \cdot\left[\int_{x=\mathrm{LSL}}^{\mu_{I}} L(x) \cdot q\left(x, \mu_{I}\right) \cdot d x\right. \\
\left.+\int_{x=\mu_{I}}^{\mathrm{USL}} L(x) \cdot q\left(x, \mu_{I}\right) \cdot d x\right] \\
+p \cdot T_{\text {out }} \cdot\left[\int_{x=\mathrm{LSL}}^{\mu_{I}} L(x) \cdot q\left(x, \mu_{O}\right) \cdot d x\right. \\
\left.+\int_{x=\mu_{I}}^{\mathrm{USL}} L(x) \cdot q\left(x, \mu_{O}\right) \cdot d x\right] .
\end{aligned}
$$

The total expected cost per production-inventory cycle is composed of setup cost, repair costs (corrective and preventive), inventory holding cost, shortage cost [15], cost of nonconforming items, and the loss cost; it is given by

$$
\begin{aligned}
& C_{\text {cycle }} \\
& \begin{array}{c}
c_{o}+c_{1} \cdot \int_{t=0}^{t_{o}} \int_{l_{1}=0}^{\infty} l_{1} \cdot g_{1}\left(l_{1}\right) \cdot f(t) \cdot d l_{1} \cdot d t \\
+c_{2} \cdot \int_{t=t_{0}}^{\infty} \int_{l_{2}=0}^{\infty} l_{2} \cdot g_{2}\left(l_{2}\right) \cdot f(t) \cdot d l_{2} \cdot d t
\end{array} \\
& +\frac{c_{i} p(p-d)}{2 d} \cdot\left[\int_{t=0}^{t_{o}} t^{2} \cdot f(t) \cdot d t+t_{o}^{2} \cdot \int_{t=t_{o}}^{\infty} f(t) \cdot d t\right] \\
& +c_{s} d \cdot \int_{t=0}^{t_{o}} \int_{l_{1}=(p-d) t / d}^{\infty}\left\{l_{1}-\frac{(p-d) t}{d}\right\} \\
& \cdot g_{1}\left(l_{1}\right) \cdot f(t) \cdot d l_{1} \cdot d t \\
& +c_{s} d \cdot \int_{t=t_{o}}^{\infty} \quad \int_{l_{2}=(p-d) t_{o} / d}^{\infty}\left\{l_{2}-\frac{(p-d) t_{o}}{d}\right\} \\
& \cdot g_{2}\left(l_{2}\right) \cdot f(t) \cdot d l_{2} \cdot d t \\
& +p \cdot T_{\mathrm{in}} \cdot\left[c_{L} \alpha_{1}+c_{U} \alpha_{2}\right]+p \cdot T_{\mathrm{out}} \cdot\left[c_{L} \beta_{1}+c_{U} \beta_{2}\right] \\
& +p \cdot T_{\mathrm{in}} \cdot\left[\int_{x=\mathrm{LSL}}^{\mu_{I}} L(x) \cdot q\left(x, \mu_{I}\right) \cdot d x\right. \\
& \left.+\int_{x=\mu_{I}}^{\mathrm{USL}} L(x) \cdot q\left(x, \mu_{I}\right) \cdot d x\right] \\
& +p \cdot T_{\mathrm{out}} \cdot\left[\int_{x=\mathrm{LSL}}^{\mu_{I}} L(x) \cdot q\left(x, \mu_{O}\right) \cdot d x\right. \\
& \left.+\int_{x=\mu_{I}}^{\mathrm{USL}} L(x) \cdot q\left(x, \mu_{O}\right) \cdot d x\right] .
\end{aligned}
$$

And the expected length of a production-inventory cycle [15] is given by

$$
\begin{aligned}
T_{\text {cycle }}= & \int_{t=0}^{t_{o}} \int_{l_{1}=0}^{(p-d) t / d}\left(\frac{p t}{d}\right) \cdot g_{1}\left(l_{1}\right) \cdot f(t) \cdot d l_{1} \cdot d t \\
& +\int_{t=0}^{t_{o}} \int_{l_{1}=(p-d) t / d}^{\infty}\left(t+l_{1}\right) \cdot g_{1}\left(l_{1}\right) \cdot f(t) \cdot d l_{1} \cdot d t \\
& +\int_{t=t_{o}}^{\infty} \int_{l_{2}=0}^{(p-d) t_{o} / d}\left(\frac{p t_{o}}{d}\right) \cdot g_{2}\left(l_{2}\right) \cdot f(t) \cdot d l_{2} \cdot d t \\
& +\int_{t=t_{o}}^{\infty} \int_{l_{2}=(p-d) t_{o} / d}^{\infty}\left(t_{o}+l_{2}\right) \cdot g_{2}\left(l_{2}\right) \cdot f(t) \cdot d l_{2} \cdot d t .
\end{aligned}
$$

From the renewal reward theorem, the average cost per unit time is given by

$$
A C\left(t_{o}, \mu_{I}\right)=\frac{C_{\text {cycle }}\left(t_{o}, \mu_{I}\right)}{T_{\text {cycle }}\left(t_{o}\right)} .
$$

Our objective is to determine the optimal production run time $t_{o}^{*}$ and the optimal process mean $\mu_{I}^{*}$ simultaneously.

Example 1. Now we solve an example by assuming the following.

Time to shift from in-control to out-of-control state is following a uniform distribution;

$$
h(\tau)= \begin{cases}\frac{1}{t}, & t \leq t_{o}, \\ \frac{1}{t_{o}}, & t \geq t_{o} .\end{cases}
$$


In order to avoid mathematical intractability, we assume exponential time to failure with failure rate $\lambda$ :

$$
f(t)=\lambda e^{-\lambda t} \text {. }
$$

Assuming exponential time to failure can be justified by noticing that in the proposed model we implement either corrective or preventive maintenance after each production run, and we assume that machine is restored to as-goodas-new condition; accordingly aging effect is minimized and process can be considered as "memory less" with time independent hazard rate $\lambda$.

Also we assume the corrective and preventive repair times to follow uniform distribution functions:

$$
\begin{array}{ll}
g_{1}\left(l_{1}\right)=\frac{1}{b_{1}}, & 0 \leq l_{1} \leq b_{1}, \\
g_{2}\left(l_{2}\right)=\frac{1}{b_{2}}, & 0 \leq l_{2} \leq b_{2} .
\end{array}
$$

And we restrict the decision variable $t_{o}$ to assume values within the following interval;

$$
0 \leq t_{o} \leq 4 .
$$

We use the following values: $p=130, d=100, c_{o}=300$, $c_{1}=1000, c_{2}=200, b_{1}=3, b_{2}=1, c_{i}=8, c_{s}=40, c_{L}=$ $30, c_{U}=20$, LSL $=250$, USL $=260, \sigma=2$, with different combinations of failure rate $\lambda$ and deterioration factor $\gamma$.

From the results shown in Table 1, we notice that, under a fixed value of failure rate $\lambda$, the optimal production run time $t_{o}^{*}$ decreases when deterioration factor $\gamma$ increases in order to reduce the out-of-control interval and hence reduce the number of nonconforming products, while the optimal process mean $\mu_{I}^{*}$ decreases and then starts to increase after a specific value of $\gamma$ which reflects the conflict between the different terms in the cost function.

On the other hand, under a fixed value of deterioration factor $\gamma$, the optimal production run time $t_{o}^{*}$ increases when failure rate $\lambda$ increases which is quite strange but can be explained by noticing that not all terms in the cost function have the same preference regarding the length of the production run time under higher failure rates. For example, from a corrective repair cost perspective, it is better to have shorter run times, while from a preventive repair cost perspective, it is better to have longer run times; and hence it is a matter of conflict resolution between the cost function terms, and accordingly the optimal run time is highly sensitive to model parameters, for example, $c_{1}, c_{2}, c_{i}$ and $c_{s}$.

And we notice reasonably that the average cost per unit time increases when any of the two parameters $(\lambda$ and $\gamma)$ is increased.

2.2. Lot Sizing, Targeting Process Mean and Standard Deviation. In what follows we consider a model to determine the optimal production run time $t_{0}^{*}$, the optimal process mean $\mu_{I}{ }^{*}$, and the optimal standard deviation $\sigma^{*}$ simultaneously. Lowering the standard deviation incurs additional cost because it requires more accurate machines which are expensive.
TABLE 1: Dependent of the optimal policy $\left(t_{o}^{*} \& \mu_{I}^{*}\right)$ on parameters $\lambda$ and $\gamma$.

\begin{tabular}{lccc}
\hline$\gamma$ & $t_{o}{ }^{*}$ & $\mu_{I}{ }^{*}$ & $\mathrm{AC}\left(t_{o}{ }^{*}, \mu_{I}{ }^{*}\right)$ \\
\hline 1.005 & \multicolumn{3}{c}{$\lambda=0.04$} \\
1.01 & 2.626 & 254.496 & 1013.53 \\
1.02 & 2.612 & 253.715 & 1109 \\
1.03 & 2.558 & 252.72 & 1428.28 \\
1.04 & 2.501 & 254.141 & 1730.86 \\
\hline \multicolumn{4}{c}{$\lambda=0.06$} \\
\hline 1.005 & 2.495 & 255.399 & 1759.87 \\
1.01 & 2.654 & 254.496 & 1083.91 \\
1.02 & 2.638 & 253.715 & 1178.08 \\
1.03 & 2.582 & 252.72 & 1492.01 \\
1.04 & 2.522 & 254.141 & 1789.71 \\
\hline \multicolumn{4}{c}{$\lambda=0.08$} \\
\hline 1.005 & 2.515 & 255.399 & 1818.25 \\
1.01 & 2.682 & 254.496 & 1152.19 \\
1.02 & 2.665 & 253.715 & 1244.87 \\
1.03 & 2.606 & 252.72 & 1553.82 \\
1.04 & 2.542 & 254.141 & 1846.79 \\
\hline
\end{tabular}

Here we are assuming that process mean is not machinerelated characteristic while the standard deviation is machine related. Moreover we assume that we have a wide range of machines available in the market varying in their accuracy (standard deviation); we need to determine the one with the optimal standard deviation which will minimize the total cost. Also we assume that all available machines are sharing the same breakdown distribution since they are all new and provided by qualified suppliers and they only differ in their accuracy. For instance, two identical CNC machines can differ in their accuracy due to different toolkits used.

The number of production cycles the new machine can serve is given by

$$
N=\frac{L_{m}}{t_{o}},
$$

where $L_{m}$ is the expected life of the machine. The standard deviation cost in a production cycle can be expressed as

$$
C_{\sigma}=\frac{K e^{\left(\sigma_{\max }-\sigma\right)}}{N}=\frac{K t_{o} e^{\left(\sigma_{\max }-\sigma\right)}}{L_{m}} .
$$

Here, $K$ is the cost incurred to reduce $\sigma$ by one unit, and $\sigma_{\max }$ is the maximum allowed value for $\sigma$.

The total cost in this case is given by

$$
\begin{aligned}
& C_{\text {cycle }} \\
& \begin{aligned}
= & c_{o}+c_{1} \cdot \int_{t=0}^{t_{o}} \int_{l_{1}=0}^{\infty} l_{1} \cdot g_{1}\left(l_{1}\right) \cdot f(t) \cdot d l_{1} \cdot d t \\
& +c_{2} \cdot \int_{t=t_{o}}^{\infty} \int_{l_{2}=0}^{\infty} l_{2} \cdot g_{2}\left(l_{2}\right) \cdot f(t) \cdot d l_{2} \cdot d t \\
& +\frac{c_{i} p(p-d)}{2 d} \cdot\left[\int_{t=0}^{t_{o}} t^{2} \cdot f(t) \cdot d t+t_{o}^{2} \cdot \int_{t=t_{o}}^{\infty} f(t) \cdot d t\right]
\end{aligned}
\end{aligned}
$$




$$
\begin{aligned}
& +c_{s} d \cdot \int_{t=0}^{t_{o}} \int_{l_{1}=(p-d) t / d}^{\infty}\left\{l_{1}-\frac{(p-d) t}{d}\right\} \cdot g_{1}\left(l_{1}\right) \cdot f(t) \\
& \cdot d l_{1} \cdot d t \\
& +c_{s} d \cdot \int_{t=t_{o}}^{\infty} \int_{l_{2}=(p-d) t_{o} / d}^{\infty}\left\{l_{2}-\frac{(p-d) t_{o}}{d}\right\} \cdot g_{2}\left(l_{2}\right) \cdot f(t) \\
& \cdot d l_{2} \cdot d t \\
& +p \cdot T_{\text {in }} \cdot\left[c_{L} \alpha_{1}+c_{U} \alpha_{2}\right]+p \cdot T_{\text {out }} \cdot\left[c_{L} \beta_{1}+c_{U} \beta_{2}\right] \\
& +p \cdot T_{\text {in }} \cdot\left[\int_{x=\mathrm{LSL}}^{\mu_{I}} L(x) \cdot q\left(x, \mu_{I}\right) \cdot d x\right. \\
& \left.+\int_{x=\mu_{I}}^{\mathrm{USL}} L(x) \cdot q\left(x, \mu_{I}\right) \cdot d x\right] \\
& +p \cdot T_{\text {out }} \cdot\left[\int_{x=\mathrm{LSL}}^{\mu_{I}} L(x) \cdot q\left(x, \mu_{O}\right) \cdot d x\right. \\
& \left.\quad+\int_{x=\mu_{I}}^{\mathrm{USL}} L(x) \cdot q\left(x, \mu_{O}\right) \cdot d x\right] \\
& +\frac{K t_{o} e^{\left(\sigma_{\max }-\sigma\right)}}{L_{m}} \cdot
\end{aligned}
$$

And the cycle length is given by (9).

And again by the renewal theory, the average cost per unit time is given by

$$
\mathrm{AC}\left(t_{o}, \mu_{I}, \sigma\right)=\frac{C_{\text {cycle }}\left(t_{o}, \mu_{I}, \sigma\right)}{T_{\text {cycle }}\left(t_{o}\right)} .
$$

Example 2. Now we solve an example to find the optimal production cycle length, the optimal process mean, and the optimal process standard deviation simultaneously. We set $K=5000, L_{m}=500, \sigma_{\max }=3$ with different combinations of failure rate $\lambda$ and deterioration factor $\gamma$, and the other parameters are same as in Example 1. And we restrict the decision variables $t_{o}$ and $\sigma$ to assume values within the following intervals: $0 \leq t_{o} \leq 4,0 \leq \sigma \leq \sigma_{\max }$.

From the results shown in Table 2, we notice that, under a fixed value of $\lambda$,

(a) $t_{o}^{*}$ decreases when $\gamma$ is increased in order to reduce nonconformities by reducing the out of control period;

(b) $\mu_{I}^{*}$ decreases then starts to increase after a specific value of $\gamma$ due to the conflict between the terms in the cost function;

(c) $\sigma^{*}$ increases then starts to decrease after a specific value of $\gamma$.

On the other hand, under a fixed value of $\gamma$ we notice the following:
(a) $t_{o}^{*}$ increases when $\lambda$ is increased;
(b) $\mu_{I}^{*}$ almost does not change when $\lambda$ is increased;
(c) $\sigma^{*}$ increases when $\lambda$ is increased.

And finally we notice that the average cost per unit time increases when any of the two parameters $(\lambda$ and $\gamma)$ is increased.
TABLe 2: Dependent of the optimal policy $\left(t_{o}^{*}, \mu_{I}^{*}, \sigma^{*}\right)$ on parameters $\lambda$ and $\gamma$.

\begin{tabular}{lcccc}
\hline$\gamma$ & $t_{o}^{*}$ & $\mu_{I}^{*}$ & $\sigma^{*}$ & $\mathrm{AC}\left(t_{o}^{*}, \mu_{I}^{*}, \sigma^{*}\right)$ \\
\hline 1.005 & 2.575 & 254.254 & 1.464 & 1119.39 \\
1.01 & 2.558 & 253.476 & 1.483 & 1226.58 \\
1.02 & 2.499 & 252.537 & 1.581 & 1574.04 \\
1.03 & 2.454 & 254.031 & 2.319 & 1880.18 \\
1.04 & 2.440 & 255.392 & 1.941 & 1914.69 \\
\hline \multicolumn{5}{c}{$\lambda=0.06$} \\
\hline 1.005 & 2.581 & 254.263 & 1.480 & 1194.87 \\
1.01 & 2.563 & 253.486 & 1.502 & 1300.15 \\
1.02 & 2.503 & 252.558 & 1.625 & 1641.17 \\
1.03 & 2.464 & 254.02 & 2.347 & 1939.33 \\
1.04 & 2.444 & 255.395 & 1.961 & 1974.51 \\
\hline \multicolumn{5}{c}{$\lambda=0.08$} \\
\hline 1.005 & 2.584 & 254.272 & 1.496 & 1268.16 \\
1.01 & 2.566 & 253.496 & 1.521 & 1371.57 \\
1.02 & 2.507 & 252.578 & 1.668 & 1706.18 \\
1.03 & 2.473 & 254.008 & 2.375 & 1996.69 \\
1.04 & 2.448 & 255.397 & 1.982 & 2032.53 \\
\hline
\end{tabular}

\section{Conclusion}

In this paper, we have investigated the joint effect of process deterioration and machine breakdown on production lotsizing and process-targeting decisions. The model is formulated under general failure and corrective repair times, while numerical examples are solved under exponential failure time and uniform repair (preventive and corrective) time distributions. We have considered two models: the first one for determining the optimal production cycle length $t_{o}^{*}$ and the optimal process mean $\mu_{I}^{*}$ simultaneously, while in the second model we introduce a new cost $C_{\sigma}$ resulting from lowering the process standard deviation $\sigma$; accordingly we determine the optimal standard deviation $\sigma^{*}$ in addition to $t_{o}^{*}$ and $\mu_{I}^{*}$. In future research, deterioration can be considered to happen gradually following some pattern (e.g., linear or exponential) rather than being sudden.

\section{Notations}

$t$ : $\quad$ Nonenegative random variable denoting time to machine breakdown

$f(t)$ : Time to breakdown probability density function pdf

$\lambda: \quad$ Failure rate (parameter for $f(t)$ ) when it's exponentially distributed

$\tau$ : $\quad$ Random variable denoting the time taken by the machine to shift from "in-control" state to "out-of-control" state

$h(\tau)$ : p.d.f. of the shift time (from in-control to out-of-control state)

$t_{o}$ : Production cycle length 
$l_{1}$ : $\quad$ Nonenegative random variable denoting corrective repair time

$g_{1}\left(l_{1}\right)$ : Corrective repair time probability density function p.d.f.

$b_{1}$ : The upper bound on $l_{1}$ when it is uniformly distributed

$l_{2}$ : $\quad$ None-negative random variable denoting preventive repair time

$g_{2}\left(l_{2}\right)$ : Preventive repair time probability density function p.d.f.

$b_{2}$ : The upper bound on $l_{2}$ when it's uniformly distributed

$T_{\text {in }}$ : $\quad$ The expected time while process is in-control

$T_{\text {out }}$ : The expected time while process is out of control

$d(>0)$ : Demand rate

$p(>d)$ : Production rate

$c_{0}(>0)$ : Set up cost for each production run

$c_{1}(>0)$ : Corrective repair cost per unit time

$c_{2}\left(<c_{1}\right)$ : Preventive repair cost per unit time

$c_{i}(>0)$ : Inventory holding cost per unit product per unit time

$c_{s}(>0)$ : Shortage penalty cost per unit product

$c_{L}(>0)$ : Cost incurred due to production of a nonconforming item with $X \leq \mathrm{LSL}$

$c_{U}(>0)$ : Cost incurred due to production of a nonconforming item with $X \geq$ USL

$C_{N}$ : Total cost of nonconforming items per cycle

$L(x)$ : Taguchi loss function

$C_{\text {Loss }}: \quad$ Total loss cost per cycle

$C_{\text {cycle: }}$ Expected total cost per production-inventory cycle

$T_{\text {cycle: }} \quad$ Expected length of a production-inventory cycle

AC: $\quad$ The average cost per unit time

$C_{\sigma}$ : $\quad$ Cost of process standard deviation $\sigma$

$N$ : $\quad$ Expected number of production cycles a new machine can serve

$L_{m}$ : $\quad$ Expected life of the machine

$K$ : $\quad$ The cost incurred to reduce $\sigma$ by one unit

$\sigma_{\max }: \quad$ The maximum allowed value for $\sigma$

$X: \quad$ Random variable denoting the quality characteristic of process under consideration

$q(x, \mu): \quad$ p.d.f. of the quality characteristic $X$

$\mu_{I}: \quad$ In-control process mean

$\mu_{O}: \quad$ Out-of-control process mean

$\gamma: \quad$ Process deterioration factor

$\sigma: \quad$ Process standard deviation

LSL: Lower specification limit on quality characteristic $X$

USL: Upper specification limit on quality characteristic $X$

$\alpha_{1}$ : $\quad$ Proportion of nonconforming while process is in-control with $X \leq \mathrm{LSL}$

$\alpha_{2} \quad$ Proportion of nonconforming while process is in-control with $X \geq \mathrm{USL}$

$\beta_{1}$ : $\quad$ Proportion of nonconforming while process is out-of-control with $X \leq \mathrm{LSL}$ $\beta_{2}$ : Proportion of nonconforming while process is out-of-control with $X \geq \mathrm{USL}$.

\section{References}

[1] J. S. Dagpunar, "The effect of minimal repairs on economic lot-sizing," Microelectronics Reliability, vol. 37, no. 3, pp. 417419, 1996.

[2] H. Kuhn, "A dynamic lot sizing model with exponential machine breakdowns," European Journal of Operational Research, vol. 100, no. 3, pp. 514-536, 1997.

[3] M. Ben-Daya, "The economic production lot-sizing problem with imperfect production processes and imperfect maintenance," International Journal of Production Economics, vol. 76, no. 3, pp. 257-264, 2002.

[4] K. J. Chung, "Approximations to production lot sizing with machine breakdowns," Computers and Operations Research, vol. 30, no. 10, pp. 1499-1507, 2003.

[5] B. C. Giri and T. Dohi, "Optimal lot sizing for an unreliable production system based on net present value approach," International Journal of Production Economics, vol. 92, no. 2, pp. 157-167, 2004.

[6] B. C. Giri and W. Y. Yun, "Optimal lot sizing for an unreliable production system under partial backlogging and at most two failures in a production cycle," International Journal of Production Economics, vol. 95, no. 2, pp. 229-243, 2005.

[7] C. C. Hsieh and Z. Z. Lee, "Joint determination of production run length and number of standbys in a deteriorating production process," European Journal of Operational Research, vol. 162, no. 2, pp. 359-371, 2005.

[8] M. Y. Jaber, "Lot sizing for an imperfect production process with quality corrective interruptions and improvements, and reduction in setups," Computers and Industrial Engineering, vol. 51, no. 4, pp. 781-790, 2006.

[9] S. W. Chiu, C. K. Ting, and Y. S. P. Chiu, "Optimal production lot sizing with rework, scrap rate, and service level constraint," Mathematical and Computer Modelling, vol. 46, no. 3-4, pp. 535-549, 2007.

[10] S. El-Ferik, "Economic production lot-sizing for an unreliable machine under imperfect age-based maintenance policy," European Journal of Operational Research, vol. 186, no. 1, pp. 150-163, 2008.

[11] A. Chelbi, N. Rezg, and M. Radhoui, "Simultaneous determination of production lot size and preventive maintenance schedule for unreliable production system producing conforming and nonconforming units," Journal of Quality in Maintenance Engineering, vol. 14, no. 2, pp. 161-176, 2008.

[12] T. Chakraborty, B. C. Giri, and K. S. Chaudhuri, "Production lot sizing with process deterioration and machine breakdown under inspection schedule," Omega, vol. 37 , no. 2, pp. 257271, 2009.

[13] D. W. Pentico, M. J. Drake, and C. Toews, "The deterministic EPQ with partial backordering: a new approach," Omega, vol. 37, no. 3, pp. 624-636, 2009.

[14] F. Hu and Q. Zong, "Optimal production run time for a deteriorating production system under an extended inspection policy," European Journal of Operational Research, vol. 196, no. 3, pp. 979-986, 2009.

[15] T. Chakraborty, B. C. Giri, and K. S. Chaudhuri, "Production lot sizing with process deterioration and machine breakdown," European Journal of Operational Research, vol. 185, no. 2, pp. 606-618, 2008. 

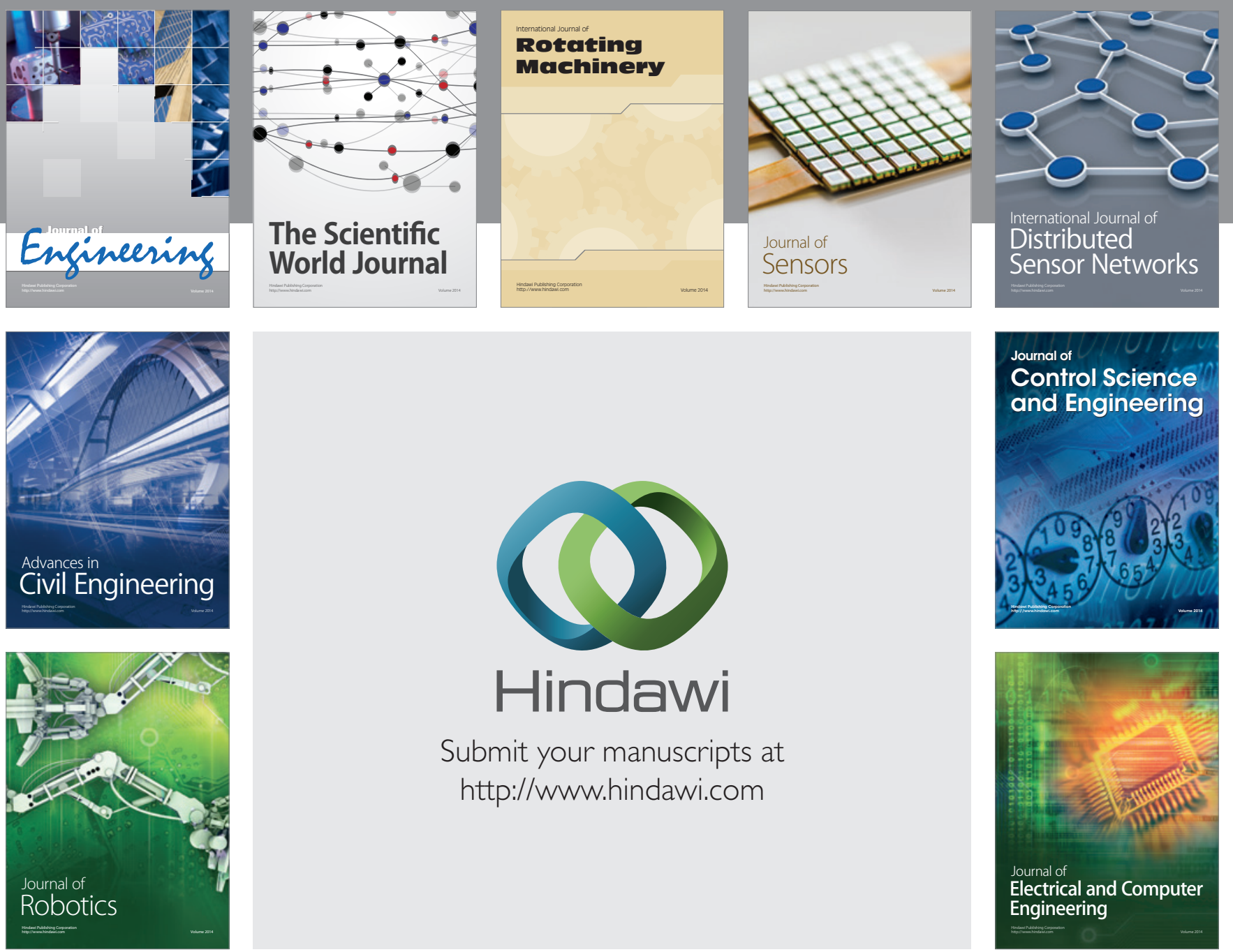

Submit your manuscripts at

http://www.hindawi.com
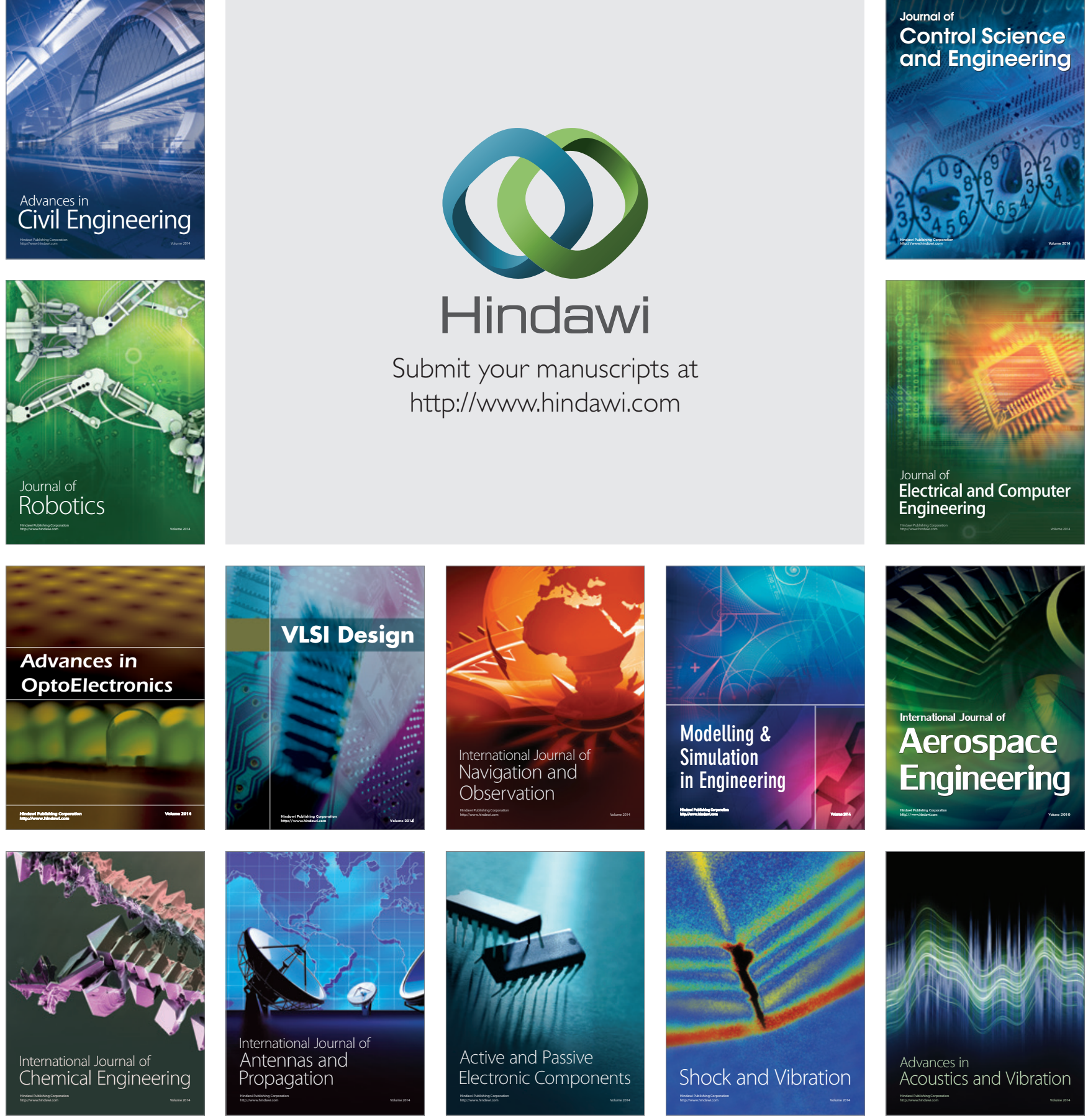